\title{
Observations of supershells in the interstellar medium of nearby galaxies
}

\author{
Elias Brinks ${ }^{1}$, Ioannis Bagetakos ${ }^{1}$, Fabian Walter ${ }^{2}$ and Erwin de Blok ${ }^{3}$ \\ ${ }^{1}$ Centre for Astrophysics Research, University of Hertfordshire, UK \\ ${ }^{2}$ Max-Planck-Institut für Astronomie, Heidelberg, Germany \\ ${ }^{3} \mathrm{MSSO}$, Australian National University, Canberra, Australia
}

\begin{abstract}
HI observations at sufficiently high spatial and velocity resolution have revealed a wealth of structures such as shells and bubbles in the ISM of late-type galaxies. These structures are filled with metal-enriched, coronal gas from SNe which, through overpressure, powers their expansion. Material swept up by these expanding shells can go "critical" and form subsequent (secondary or propagating) star formation. Shells that grow larger than the thickness of the gas layer will blow out of the disk, spilling enriched material into the halo (or in the case of violent starbursts, the Intergalactic Medium). We review what has been achieved to date and present some first results of a major project based on THINGS (The HI Nearby Galaxy Survey), which aims to extend studies of the ISM in galaxies to 34 nearby systems $(<10 \mathrm{Mpc})$, all observed to the same exacting standards (resolution $6^{\prime \prime} \times 5 \mathrm{~km} \mathrm{~s}^{-1}$, or better; typical detection threshold of $\sim 5 \times 10^{19}$ atom $\mathrm{cm}^{-2}$ ).
\end{abstract}

Keywords. galaxies: ISM — ISM: bubbles — ISM: structure — radio lines: ISM

\section{Introduction}

The formation of massive stars in a gas-rich spiral or dwarf galaxy has a dramatic effect on the surrounding Interstellar Medium (ISM). Newly formed massive stars $\left(\mathrm{M}>8 \mathrm{M}_{\odot}\right)$ will have a major impact, first of all through their ionizing flux and stellar winds and, when they eventually have exhausted their fuel supply, as supernovae (SNe). Because massive stars usually form in clusters or associations a large amount of mechanical energy is dumped into the ISM, within a small volume and within a short time span, creating large-scale structures. These go by various descriptions, bubbles, shells or holes, based in part on their appearance at the different wavelengths at which they have been studied. Following Chu et al. (2004), bubbles and shells are created by single stars, superbubbles and supergiant shells require multiple stars for their formation. If they grow larger than the scale height of the gas disk, superbubbles break out into the halo. Bubbles and shells appear as "holes" in maps of neutral hydrogen, hence their name.

The study of shells and supershells is relevant to many areas of galaxy research. Supergiant shells are linked to superbubble blow out and might lie at the origin of a galactic fountain (Bregman 1980). If shells do indeed break out of the disk, the halo or in extreme cases even the intergalactic medium can be enriched by metals produced in the massive stars before they exploded as SNe (Tenorio-Tagle 2000). Actively star forming dwarf irregular galaxies are most prone to mass loss (Mac Low \& Ferrara 1999; Silich \& Tenorio-Tagle 2001). Given that in the currently accepted $\Lambda$ CDM cosmology dwarf galaxies dominated at large look-back times, this would explain the rapid enrichment and mixing of heavy elements shortly after the first galaxies were formed. 
Having said that, $\mathrm{X}$-ray observations of actively star forming dwarfs show that it is exceedingly difficult for material to escape, even from dwarf galaxies (Ott et al. 2005a, b). Material can be transported upwards into the dark matter dominated halo, though, increasing the cross section for objects lying between the observer and distant QSOs and going some way to explaining the frequency of Damped Ly $\alpha$ features and associated metal lines as a function of redshift.

On a more local scale, expanding shells compress material on their rims which in turn can reach conditions conducive to star formation (Tenorio-Tagle et al. 2005). Examples for this can be found in the LMC where CO observations point at the presence of molecular clouds on the rims of several supergiant shells (Yamaguchi et al. 2001; Fukui, this volume). The Magellanic Clouds are not the only example. Leroy et al. (2006; see also Blitz, this volume) show similar such accumulations of molecular gas on the interface between two neighbouring shells in IC 10 which seemingly are running into each other. In other words, shells could be driving self-regulating (propagating or stochastic) star formation (Elmegreen et al. 2002). On yet smaller scales, the processes leading to shells and supergiant shells provide energy input on the largest turbulent scales which then cascade down to ever smaller scales.

Shells are useful as they provide an independent means to gauge the scale height of the neutral ISM (Brinks et al. 2002). As Oey \& García-Segura (2004) have shown, they can be used as barometers as well. And finally, the energy input from $\mathrm{SNe}$ in the form of expanding shells provides positive feedback as it raises the velocity dispersion in the gas, shutting off further star formation until such time as the gas has cooled down, in a dynamical sense, allowing SF to recommence. This feedback loop is likely what causes the ISM to have a one dimensional velocity dispersion of $6-10 \mathrm{~km} \mathrm{~s}^{-1}$ irrespective of galaxy type (e.g., Dib et al. 2006).

\section{Observations of supergiant shells}

Although the first structures we now describe as supergiant shells were first recognized by Hindman (1967) in the SMC, it was the seminal work by Heiles (1984) in the Milky Way galaxy which gave prominence to this field. In general, observations in the $21-\mathrm{cm}$ line of neutral hydrogen have proven crucial. HI nowadays is easy to observe and aperture synthesis interferometers can reach linear resolutions of $15-50 \mathrm{pc}$ in the Local Group. Maps of the HI surface brightness show a wealth of structure and, assuming that the HI is optically thin, are a direct measure of the surface density. Moreover, as we're dealing with a spectral line, the expansion velocities of giant shells can be measured, allowing estimates to be derived of the age of the structures and energies typically required to produce them. To date, detailed observations of over a dozen galaxies have been published (see Table 1 for a list of published studies of (super)giant shells based on HI observations).

Interestingly, superbubbles are seen in molecular gas as well. I am aware of three galaxies where they have been seen in the line of ${ }^{12}$ CO: M 82 (Weiß et al. 1999), NGC4666 (Walter et al. 2004), and NGC 253 (Sakamoto et al. 2006). In the latter galaxy there is a tentative detection in even denser clouds as traced by $\mathrm{NH}_{3}$ (Ott et al. 2005c).

Giant and supergiant shells have been seen at other wavelengths as well, of course, notably in $\mathrm{H} \alpha$. Some recent papers are those by Relaño \& Beckman (2005), Ambrocio-Cruz et al. (2004), and Valdez-Gutiérrez et al. (2002), all reporting Fabry-Perot observations. A lot of the earlier work was based on echelle spectra (cf. early papers on the LMC by Meaburn 1980, and on the 30 Dor region by Chu \& Kennicutt 1994).

Most recently, observations with the Spitzer Space Telescope have revealed a stunning level of detailed structure in several nearby galaxies, notably the maps at $8 \mu \mathrm{m}$ and $24 \mu \mathrm{m}$ 
Table 1. Published studies of (super)giant shells based on HI observations

\begin{tabular}{|c|c|c|c|}
\hline Galaxy & authors & Galaxy & authors \\
\hline Milky Way & Heiles (1984) & IC 10 & Wilcots \& Miller (1998) \\
\hline LMC & Kim et al. (2003) & IC 1613 & Silich et al. (2006) \\
\hline $\mathrm{SMC}$ & Stanimirović et al. (1999) & IC 2574 & Walter \& Brinks (1999) \\
\hline M 31 & Brinks \& Bajaja (1986) & Holmberg I & Ott et al. (2001) \\
\hline M 33 & Deul \& den Hartog (1990) & Holmberg II & Puche et al. (1992) \\
\hline M 101 & Kamphuis et al. (1991) & DDO 43 & Simpson et al. (2005b) \\
\hline NGC 1569 & Mühle et al. (2005) & DDO 47 & Walter \& Brinks (2001) \\
\hline NGC 6946 & Boomsma et al. (2004) & DDO 88 & Simpson et al. (2005a) \\
\hline
\end{tabular}

tracing the emission of PAHs and warm dust, respectively (see, e.g., the recent study of the supergiant shell in IC 2574 by Cannon et al. 2005).

These large, coherent structures in the ISM of galaxies have been interpreted in terms of a "standard model" (see Oey, this volume, for an overview) for which there is ample support. X-rays have been detected from the coronal gas filling supergiant shells (Townsley et al. 2006, and references therein), many such shells have associated $\mathrm{H} \alpha$ emission in the form of expanding shells (Relaño, this volume). Often a remnant young stellar cluster found to be co-spatial with the shell and UV emission is correlated with these objects (Stewart et al. 2000).

On the theoretical side, hydrodynamical and MHD simulations are able to reproduce the observed structures based on energetics commensurate with a SN origin (Oey, this volume). Also, the observed superbubble size distribution fits predictions based on the HII region luminosity function (Oey \& Clarke 1997).

Notwithstanding the consensus that star clusters through the dimise of the most massive stars as SNe power supergiant shells, several alternative explanations have been put forward to explain at least some of them, primarily those which are found where star formation is rather low key or for those cases for which the energy requirement to create the structure far surpasses what can be delivered by even a super star cluster (SSC). Among the mechanisms proposed is infall of high velocity clouds (Tenorio-Tagle et al. 1987), ram pressure enlarging embryonic shells (Bureau \& Carignan 2002), and turbulence, coupled with cooling and gravitational instability (Wada et al. 2000; Dib \& Burkert 2005).

Based on observations obtained thus far, giant and supergiant shells have been found with diameters ranging from the resolution limit of the telescopes employed (20-100 pc, typically, for nearby galaxies) to kpc size. Energy requirements range from the kinetic energy deposited in the ISM by a single SN to that of several hundred SNe. Estimated ages for the shells can reach of order $10^{8} \mathrm{yr}$ for the larger structures.

Walter \& Brinks (1999) made a comparison between four objects, two spirals, M31 and M 33, and two dwarf irregular galaxies, Holmberg II and IC 2574, and reached the following important conclusions. First of all the energy deposited by a young star cluster or stellar association is, to first order, independent of the host galaxy. Secondly, dwarf galaxies have a shallower gravitational potential. This, combined with the fact that they rotate as a solid body over most of their HI disk, results in the counterintuitive result that shells in dwarf galaxies can grow to larger sizes than in large spiral galaxies. Because the neutral gas layer in a spiral galaxy is thinner, in absolute size, than that of a typical dwarf this means that an expanding shell will blow out of the disk once it reaches a diameter which is of order the full width to half density thickness of the gas layer. 


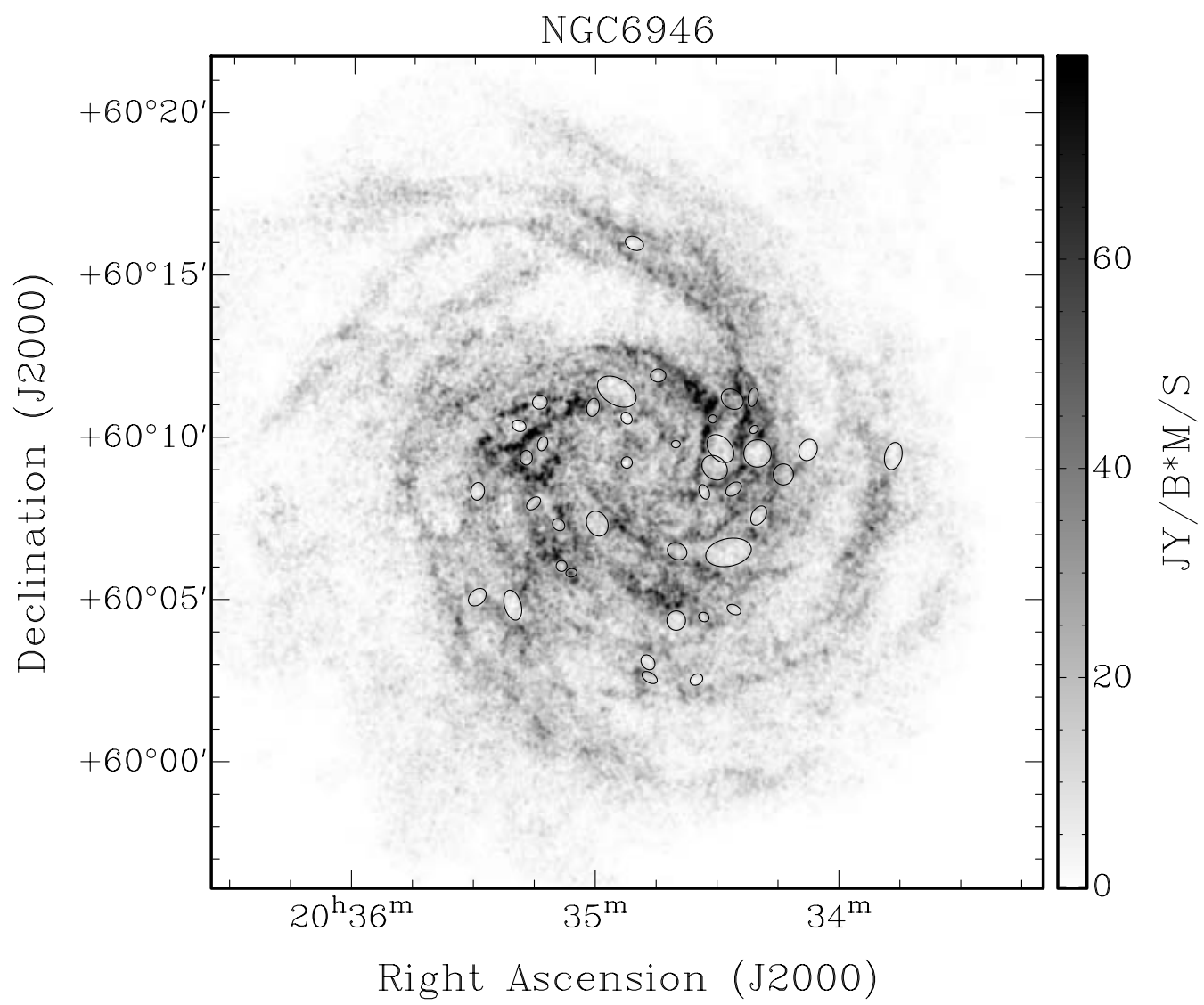

Figure 1. HI surface brightness map of NGC 6946. The ellipses mark the locations of some 40 well-defined HI holes.

\section{Future projects}

Those galaxies which have been observed at high enough spatial and velocity resolution plus sufficient sensitivity to allow the small scale structure of the ISM to be studied are a rather eclectic collection (see Table 1). In order to put the tentative conclusions reached by Walter \& Brinks (1999) on a much firmer footing, we initiated an investigation of the galaxies which form part of THINGS, The HI Nearby Galaxy Survey (Walter et al. 2005). THINGS targets 34 nearby $(<10 \mathrm{Mpc})$ late-type spiral and irregular galaxies, covering a range of metallicities and star formation rates. The resolution of the maps obtained as part of THINGS is $6^{\prime \prime} \times 5 \mathrm{~km} \mathrm{~s}^{-1}$, or better; typical detection thresholds are

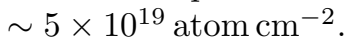

The galaxy M 81 was recently analysed by us (Bagetakos et al., this volume) and several more systems are currently being worked on. Fig. 1 presents the HI surface density map of NGC 6946 showing of order 40 well-defined HI holes. Some HI holes can readily be identified in the HI surface brightness map, others stand out when going through the 


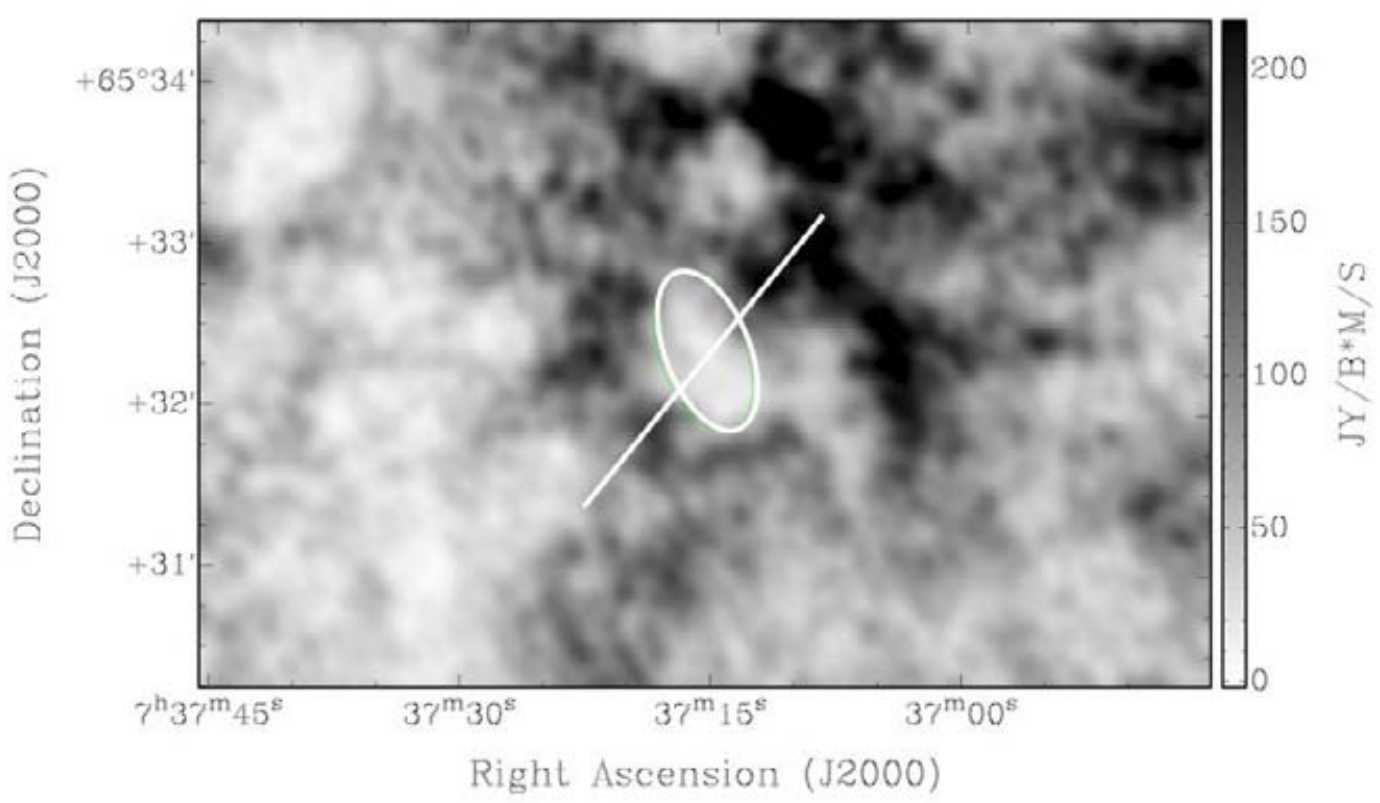

Figure 2. HI surface brightness map of a $4^{\prime} \times 5^{\prime}$ area of NGC 2403 , showing a wealth of structure. The location of one HI hole is indicated by the ellipse.

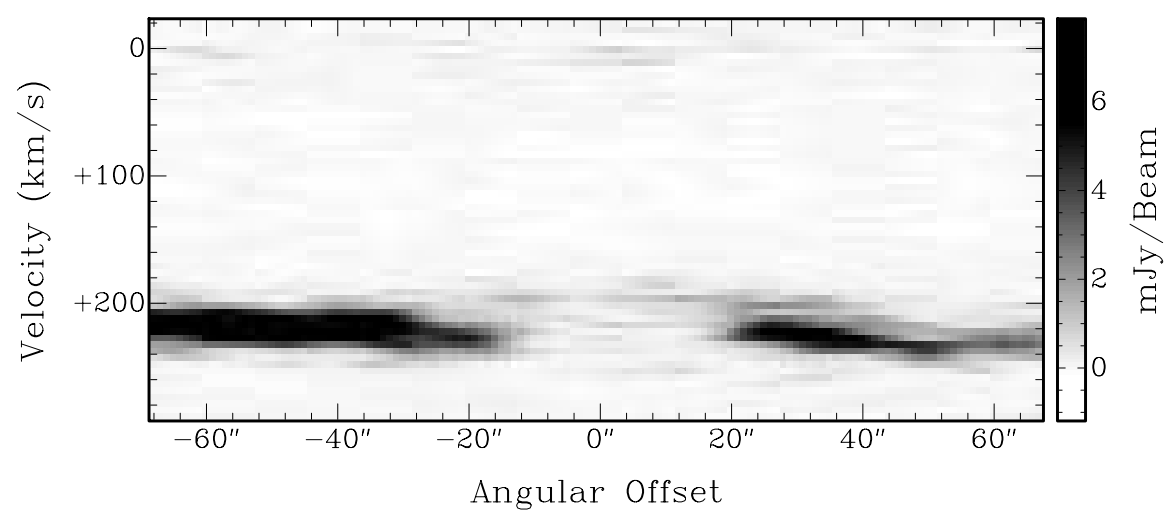

Figure 3. Position-velocity map along the line cutting through the HI hole shown in Fig. 2. Virtually no HI is left at the location of the hole, suggesting that blow out has occurred.

individual velocity channels or upon inspection of the HI data cube in position-velocity space. Despite efforts by various authors to devise an automated procedure to identify HI holes (see Ehlerová \& Palouš 2005, and references therein), no single method has emerged yet that manages to match the human eye/brain in picking out these structures. Once a potential HI hole has been identified, a position-velocity cut readily confirms its reality, as illustrated in Figs. 2 and 3 which shows a typical example of an HI hole in yet another object, NGC 2403. 
Work has now started to analyse in a consistent and uniform manner all galaxies within THINGS (Bagetakos et al., this volume). The idea is to prepare for each galaxy a catalogue of HI holes. The results for each galaxy will be compared in order to investigate how the diameter distribution of HI holes, their ages and energy requirements correlate with Hubble type, metallicity, star formation rate, etc. The structures found in HI will be compared with mid-IR data from the Spitzer SINGS survey, UV maps obtained with GALEX, and optical broad-- and narrow-band ( $\mathrm{H} \alpha)$ imaging.

\section{Summary}

Maps in the line of HI at sufficiently high linear resolution (100 pc or better) are dominated by giant and supergiant shells and filaments. Many of the structures are coherent and expand. The dimensions, energetics and general characteristics are compatible with a SN origin. The energy input of a young star cluster is to first order independent of Hubble type. Dwarf galaxies have as a result larger diameter shells. Expanding shells are not only seen in $\mathrm{HI}$ but also in $\mathrm{H} \alpha$ and molecular line emission $\left(\mathrm{CO}, \mathrm{NH}_{3}\right)$. Sites of secondary SF can be seen on the rims of some shells and CO emission is detected on the interface where neighbouring shells run into each other compressing, between them, the surrounding ISM. Shells are at the top of the turbulence cascade and some of the smaller shells could be turbulence driven.

Most of the results and ideas we have about giant and supergiant shells are based on only a handful of galaxies, many of them of the dwarf variety and some clearly pathological. Given the importance of shells, from fields as diverse as the enrichment of the IGM in the early universe to star formation triggered by pressure-driven, expanding bubbles, a major project has been conceived by us, THINGS, which will produce maps of the highest quality of the ISM of 34 nearby galaxies. Eventually, THINGS will firm up many of the tentative results arrived at thus far and go a long way to addressing some of the key questions of this fascinating field.

\section{References}

Ambrocio-Cruz, P., Laval, A., Rosado, M., Georgelin, Y. P., Marcelin, M., Comeron, F., Delmotte, N. \& Viale, A. 2004, AJ 127, 2145

Boomsma, R., van der Hulst, T., Oosterloo, T., Fraternali, F. \& Sancisi, R. 2004, in: P.-A. Duc, J. Braine \& E. Brinks (eds.), Proc. IAU Symp. No. 217 (San Francisco: ASP), p.142

Bregman, J. N. 1980, ApJ 236, 577

Brinks, E. \& Bajaja, E. 1986, A\&A 169, 14

Brinks, E., Walter, F. \& Ott, J. 2002, ASP-CS 275, 57

Bureau, M. \& Carignan, C. 2002, AJ 123, 1316

Cannon, J. M., et al. 2005, ApJ 630, L37

Chu, Y.-H. \& Kennicutt, R. C., Jr. 1994, ApJ 425, 720

Chu, Y.-H., Guerrero, M. A. \& Gruendl, R. A. 2004, in: E. J. Alfaro, E. Pérez \& J. Franco (eds.), How Does the Galaxy Work? A Galactic Tertulia with Don Cox and Ron Reynolds, ApESSS (Kluwer, Dordrecht), vol 315, p. 165

Deul, E. R. \& den Hartog, R. H. 1990, A\&广A 229, 362

Dib, S. \& Burkert, A. 2005, ApJ 630, 238

Dib, S., Bell, E. \& Burkert, A. 2006, ApJ 638, 797

Ehlerová, S. \& Palouš, J. 2005, A\&A A 437, 101

Elmegreen, B. G., Palouš, J. \& Ehlerová, S. 2002, MNRAS 334, 693

Heiles, C. 1984, ApJS 55, 585

Hindman, J. V. 1967, AuJPh 20, 147

Kamphuis, J., Sancisi, R. \& van der Hulst, T. 1991, A\&SA 244, L29 
Kim, S., Staveley-Smith, L., Dopita, M. A., Sault, R. J., Freeman, K. C., Lee, Y. \& Chu, Y.-H. 2003, ApJS 148, 473

Leroy, A., Bolatto, A., Walter, F. \& Blitz, L. 2006, ApJ 643, 825

Mac Low, M.-M. \& Ferrara, A. 1999, ApJ 513, 142

Meaburn, J. 1980, MNRAS 192, 365

Mühle, S., Klein, U., Wilcots, E. M. \& Hüttemeister, S. 2005, AJ 130, 524 (Erratum: 2006, AJ $132,443)$

Oey, M. S. \& Clarke, C. J. 1997, MNRAS 289, 570

Oey, M. S. \& García-Segura, G. 2004, ApJ 613, 302

Ott, J., Walter, F. \& Brinks, E. 2005a, MNRAS 358, 1423

Ott, J., Walter, F. \& Brinks, E. 2005b, MNRAS 358, 1453

Ott, J., Weiß, A., Henkel, Ch. \& Walter, F. 2005c, ApJ 629, 767

Ott, J., Walter, F., Brinks, E., Van Dyk, S. D., Dirsch, B. \& Klein, U. 2001, AJ 122, 3070

Puche, D., Westpfahl, D., Brinks, E. \& Roy, J.-R. 1992, AJ 103, 1841

Relaño, M. \& Beckman, J. E. 2005, A\& $A$ 430, 911

Sakamoto, K., Ho, P. T. P., Iono, D., Keto, E. R., Mao, R.-Q., Matsushita, S., Peck, A. B., Wiedner, M. C., Wilner, D. J. \& Zhao, J.-H. 2006, ApJ 636, 685

Silich, S. \& Tenorio-Tagle, G. 2001, ApJ 552, 91

Silich, S., Lozinskaya, T., Moiseev, A., Podorvanuk, N., Rosado, M., Borissova, J., ValdezGutiérrez, M. 2006, A\&\&A 448, 123

Simpson, C. E., Hunter, D. A. \& Knezek, P. M. 2005a, AJ 129, 160

Simpson, C. E., Hunter, D. A. \& Nordgren, T. E. 2005b, AJ 130, 1049

Stanimirović, S., Staveley-Smith, L., Dickey, J. M., Sault, R. J. \& Snowden, S. L. 1999, MNRAS 302,417

Stewart, S. G., et al. 2000, ApJ 529, 201

Tenorio-Tagle, G. 2000, NewAR 44, 365

Tenorio-Tagle, G., Franco, J., Bodenheimer, P. \& Rozyczka, M. 1987, A\&̈A 179, 219

Tenorio-Tagle, G., Silich, S., Rodríguez-González, A. \& Muñoz-Tuñón, C. 2005, ApJ 628, L13

Townsley, L. K., Broos, P. S., Feigelson, E. D., Brandl, B. R., Chu, Y.-H., Garmire, G. P. \& Pavlov, G. G. 2006, $A J$ 131, 2140

Valdez-Gutiérrez, M., Rosado, M., Puerari, I., Georgiev, L., Borissova, J. \& Ambrocio-Cruz, P. 2002, AJ 124, 3157

Wada, K., Spaans, M. \& Kim, S. 2000, ApJ 540, 797

Walter, F., Dahlem, M. \& Lisenfeld, U. 2004, ApJ 606, 258

Walter, F. \& Brinks, E. 1999, AJ 118, 273

Walter, F. \& Brinks, E. 2001, AJ 121, 3026

Walter, F., Brinks, E., de Blok, W. J. G., Thornley, M. D. \& Kennicutt, R. C. 2005, ASP-CS 331,269

Weiß, A., Walter, F., Neininger, N. \& Klein, U. 1999, A\&̋A 345, L23

Wilcots, E. M. \& Miller, B. W. 1998, AJ 116, 2363

Yamaguchi, R., Mizuno, N., Onishi, T., Mizuno, A. \& Fukui, Y. 2001, ApJ 553, L185

\section{Discussion}

Tenorio-Tagle: A suggestion would be to look for shells also in the gaseous halos of galaxies. they would be much fainter than the ones you are detecting on disks but they are there.

BRINKS: That's indeed a good suggestion, and I hope that you will be asked on the time allocation committee to insure we will be awarded the time needed to pursue this!

MAC Low: Why do density power spectra appear to extend well beyond the supershell scale if supershells are the driver of the turbulence?

BRINKS: I have no idea! Regarding our data, we haven't yet produced any power spectra. 
OEY: I don't think I agree with your suggestion that galaxies with higher star formation show more HI shells. The opposite is seen in the Magellanic clouds: the LMC has only $1 / 4$ the number of HI shell as the SMC. I think this may be because if the SFR is high enough, then the shells will merge and shred the ISM, etc. This may also be related to the earlier question about the large extension of the power spectrum.

BRINks: Yes, I agree that the LMC looks "shredded". We will look, though, across our sample if we find trends with star formation rate, Hubble type, etc., in the hope to be able to increase our underestimate of the shells.

ZINNECKER: Is the secondary star formation on the rim of the shells as violent as that of the first star formation which gave rise to the shells?

BRINKS: This is hard to tell. We can estimate the kind of association that was responsible for creating the holes, but with what do you compare this, with a single star forming region on the rim, or with all such regions averaged over the entire rim? 